\title{
Effects of alcohol on myoclonus and somatosensory evoked potentials in dyssynergia cerebellaris myoclonica
}

\author{
Chin-Song Lu, Nai-Shin Chu
}

\begin{abstract}
Three brothers with dyssynergia cerebellaris myoclonica received alcohol to study the correlation between improvement of myoclonus and alteration in somatosensory evoked potentials (SEPs). Alcohol considerably improved myoclonus for about six hours in two patients (cases 1 and 2) but had only a mild effect in one (case 3 ). All three patients had giant cortical SEPs. The amplitudes of median N20-P25 and P25N35 components and tibial N30-P40 and P40-N50 components were considerably decreased after alcohol ingestion in two patients (cases 1 and 2) but unchanged or slightly decreased in one (case 3 ). The peak latencies of those components were not affected by alcohol. There was thus a good correlation between the suppression of myoclonus and the decrease in giant SEP amplitude.
\end{abstract}

Ramsay Hunt had observed a temporary relief of action myoclonus by alcohol in one (case 1) of six patients with dyssynergia cerebellaris myoclonica (DCM), also known as Ramsay Hunt syndrome." $\mathrm{He}$ stated "they were usually diminished by the use of alcohol, and the patient sometimes took this means of relieving their severity". In 1963, Gilbert et $a l^{2}$ also mentioned "his tremor was worsened by nervousness and anxiety but alleviated by drinking alcohol" in one (case 1) of four patients with familial myoclonus and ataxia. Nevertheless, this alcoholic remedy has rarely been reported subsequently. There was no further explanation concerning the electrophysiological mechanism underlying the effect of alcohol on myoclonus.

In 1947 Dawson first described in a myoclonic patient a pathological enhancement of cortical responses following sensory stimulation of the limbs. ${ }^{3}$ In cortical reflex myoclonus, myoclonic jerking is often evoked by the same stimulus that produces the giant somatosensory evoked potentials (SEPs). ${ }^{4}$ The size of the SEP may sometimes dissociate with reflex myoclonus, but it is generally correlated with the intensity of myoclonic activities. When therapeutic control of the myoclonus is effective there is'also a reduction in the amplitude of giant SEPs. ${ }^{47}$ Although the enhanced cortical SEP is well documented in patients with $\mathrm{DCM}^{8}{ }^{8}$ neurophysiological study on the influence of alcohol on the enlarged SEPs, which seem to relate to the severity of myoclonus, has not been reported.

On reviewing eight consecutive patients with DCM, we were surprised to find three brothers who reported that their myoclonus was improved by alcohol. To confirm their observation and to elucidate the possible site of the effect of alcohol, we studied the improvement of myoclonus and the alteration in SEPs after alcohol in these three patients.

\section{Subjects}

The subjects were three brothers aged 28 (case 1), 26 (case 2) and 24 years (case 3), respectively. The age of onset of symptoms was around 16 years. They presented with marked action myoclonus, mild cerebellar ataxia and occasional generalised seizures. The past history including birth and development was normal in all three. The parents were non-consanguineous.

The action myoclonus was the most distressing symptom which appeared initially in the lower limbs and progressively involved the upper limbs and trunk. Voluntary movements of the limbs precipitated a salvo of focal or generalised muscle jerking. In addition, they had myoclonus of the face and tongue during speech. There was rare myoclonus at complete rest, but jerks could be induced by a pinprick or tactile stimulation. Occasional falling attacks without loss of consciousness could be provoked by unexpected loud noise. They walked with a wide-based gait and moved carefully along the wall for fear of falling.

Rarely, they also experienced generalised convulsions, which occurred most often in the early morning while asleep. There were about eight seizures in patients 2 and 3 and only one in patient 1 since the onset of their symptoms. After a seizure, the myoclonus was less evident for several hours.

On examination, they were alert and were mentally normal. There was no abnormality except myoclonus, particularly action myoclonus, and slight cerebellar ataxia. The possible existence of limb dysmetria, however, was difficult to evaluate because of the accompanying severe action myoclonus. Laboratory investigations were all normal including haematology, erythrocyte sedimentation rate, electrolytes, liver and thyroid function tests, lactate, pyruvate, serology for syphilis, folate, copper and ceruloplasmin, lysosomal enzyme tests in leukocyte, CSF, immunoelectro- 
phoresis, nerve conduction velocity, electromyography, electroencephalography, CT of the brain, brain stem auditory evoked potential, pattern-reversal visual evoked potential, and muscle biopsy.

The action myoclonus was considerably improved by combined treatment with clonazepam $1.5 \mathrm{mg}$, piracetam $9 \mathrm{~g}$ and sodium valproate $600 \mathrm{mg}$ daily. They still could not execute fine movements such as writing and holding chopsticks without jerking. However, if they took several big gulps of alcohol, usually about $200 \mathrm{ml}$, their myoclonus disappeared dramatically for several hours, particularly in two patients (cases 1 and 2).

\section{Methods}

SEP recording was conducted in a quiet and semi-darkened room with the subject in a supine position. Room temperature was kept constant at $24^{\circ} \mathrm{C}$ by central air conditioning. The median nerve was stimulated with a biopolar disc electrode at the wrist with the cathode placed $2.5 \mathrm{~cm}$ proximal to the stimulation electrode. Electric shocks delivered at $2 / \mathrm{s}$ were square pulses of 0.2 ms duration with the intensity adjusted to produce minimal twitch of the thumb. The posterior tibial nerve was stimulated at the ankle with the intensity adjusted to elicit minimal flexion of the toes. Median cortical SEPs were recorded between
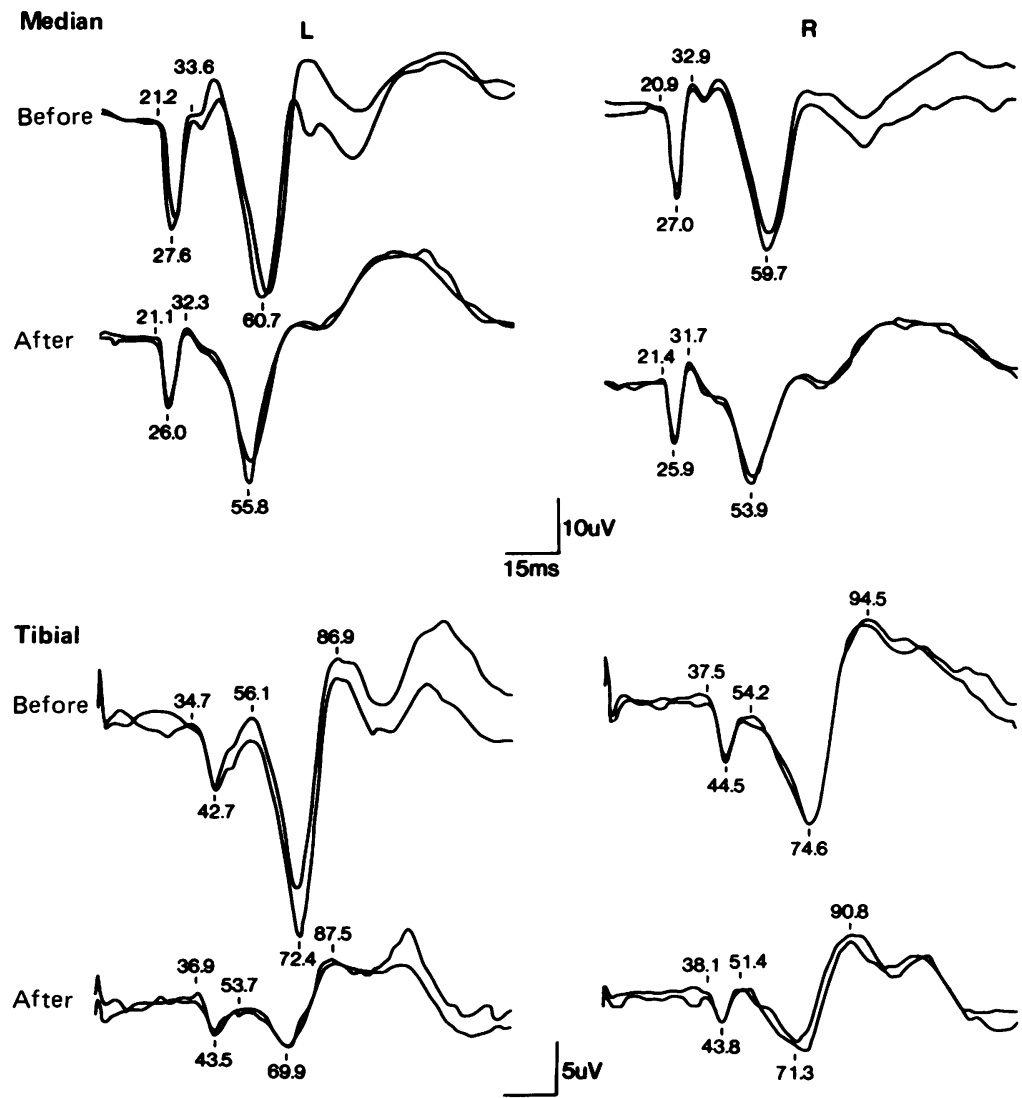<smiles>C1C2CC1C2</smiles>
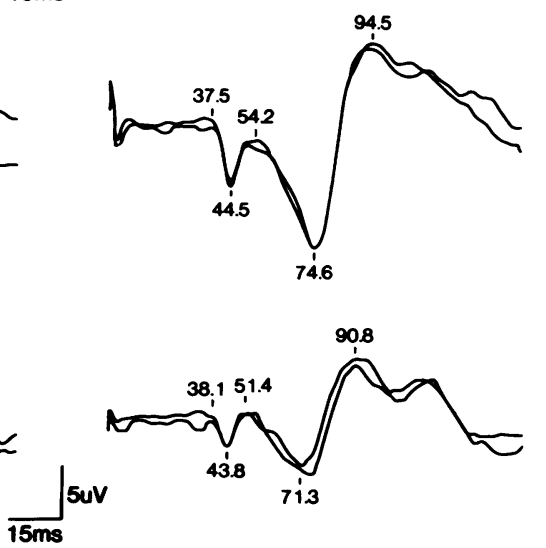

Figure Median and tibial cortical SEPs before (pre) and one hour after (post) alcohol ingestion in patient 1. The stimulation was at the beginning of the tracings. The upper calibration for median SEPs and the lower calibration for tibial SEPS. an electrode at $\mathrm{Fz}$ and an electrode over the scalp $2 \mathrm{~cm}$ posterior to $\mathrm{C} 3$ or $\mathrm{C} 4$ contralateral to the side of nerve stimulation. Tibial cortical SEPs were recorded between electrodes at $\mathrm{Fz}$ and at the midline $2 \mathrm{~cm}$ posterior to $\mathrm{Cz}$. Ground electrode was situated approximately $5 \mathrm{~cm}$ above the stimulation site. Electrode impedance was kept below $5 \mathrm{~K}$ ohms. The evoked responses were amplified with a bandpass of $20-3 \mathrm{~K} \mathrm{~Hz}$ and summed over $200-300$ responses. At least two averages were obtained to ensure reproducibility.

All medications including clonazepam, piracetam and sodium valproate were discontinued for three days. A baseline median and tibial SEP recordings were first performed. The patients were then asked to drink $300 \mathrm{ml}$ of brandy $(41 \%)$ within 30 minutes. SEPs from the median and tibial nerve stimulation were recorded within the following hour. The duration of the effect of alcohol on myoclonus was also recorded. The latency and amplitude of the median and tibial cortical SEPs were measured. Paired $t$ test was used to determine the statistical differences.

\section{Results}

Before drinking alcohol, the patients had great difficulty in walking or holding a cup because of the severe action myoclonus. About 20 minutes after alcohol ingestion, the action myoclonus disappeared in patients 1 and 2 who could then walk or pick up the cup easily. But in patient 3, the myoclonus was only mildly improved. Patient 1 complained of mild dizziness and nausea after he had finished drinking. None of the patients became intoxicated. They were alert throughout the recordings. The alcohol suppression of action myoclonus lasted six hours in patient 1 , and 5.5 hours in patient 2 . Although the action myoclonus stopped during this period, mild dysmetria was observed in finger-nose-finger tests. They still failed to walk heel-to-toe along a straight line.

All three patients had giant median and tibial cortical SEPs before drinking. However, the peak latencies were normal. The normative data from our laboratory showed that the mean (SD) amplitude of median N20-P25 and P25N35 was respectively $2.36(0.81) \mathrm{uV}$ and 1.44 $(0.85) \mathrm{uV}$. The mean (SD) amplitude of tibial N30-P40 and P40-N50 was respectively 1.26 $(0.74) \mathrm{uV}$ and $1.41(0.76) \mathrm{uV} .^{9}$ There was a marked reduction in the amplitudes of the median N20-P25 and P25-N35 and tibial N30-P40 and P40-N50 components in patient 1 (fig) and patient 2 at one hour after drinking, but these amplitudes were only slightly decreased in patient 3 . The SEP cortical components later than median N35 and tibial N50 were also.suppressed by alcohol in patients 1 and 2 but not in patient 3 . The table summarises the reduction of median and tibial cortical SEPs at one hour after alcohol ingestion. Both median and tibial cortical SEPs were similarly affected by alcohol. The reduction in the amplitude are statistically greater if the data from patients 1 and 2 are used. The cortical SEPs of patient 3 were unchanged or only 
Table Effect of alcohol on the amplitude ( $\mathrm{UV}$ ) of median and tibial cortical SEPs

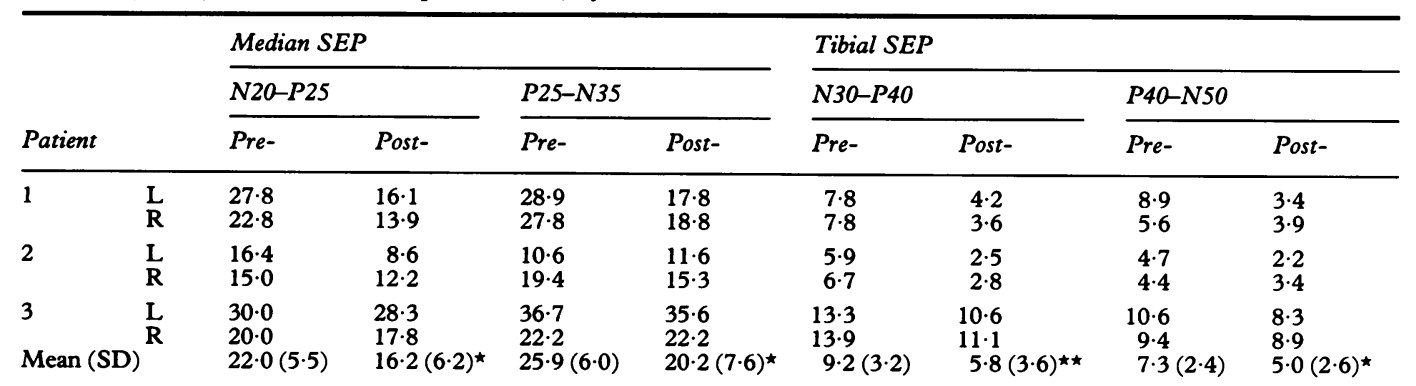

1 Abreviations: Pre- = before alcohol, Post- = one hour after alcohol, $\mathrm{L}=$ left, $\mathrm{R}=$ right.

2 Statistical significance: ${ }^{\star}=p<0.05$ and ${ }^{\star \star}=p<0.001$.

slightly decreased by alcohol. However, the peak latencies of median N20, P25 and N35 and tibial N30, P40 and N50 components were not altered by alcohol.

\section{Discussion}

Recently there has been much discussion about the nosology of Ramsay Hunt syndrome. ${ }^{10-12}$ Our patients had marked action myoclonus, progessive cerebellar ataxia and rare generalised seizures which fulfilled the triad of this syndrome. ${ }^{10}$ Meanwhile, normal lactate and pyruvate levels in serum and muscle biopsy in our patients did not support a diagnosis of mitochondrial encephalomyopathy with ragged-red fibres.

Our results show that alcohol could effectively attenuate the giant cortical SEPs in two patients who also had dramatic improvement of action myoclonus. Although the quantity of alcohol given was quite a large amount, the myoclonus disappeared 20 minutes (about $180 \mathrm{ml}$ alcohol) after they started to drink. In fact, they usually took $200 \mathrm{ml}$ of spirit which suppressed the myoclonus. The third patient who gained little benefit for myoclonus from the alcohol did not have a reduction in enhanced cortical SEPs. Thus the alcohol suppression of enhanced cortical SEPs correlated with the degree of alcohol control of the muscle jerks. The attenuation in the amplitude of cortical SEPs after alcohol ingestion has been demonstrated in humans. ${ }^{13}$ Alcohol has a similar effect on the giant SEPs in patients with DCM which has not been reported previously. Why the giant SEPs and myoclonus did not have a similar response to alcohol in the third patient is unknown. On the other hand, the selective improvement of myoclonus in our two patients with DCM needs further study.

The median N20-P25 and P25-N35 and tibial N30-P40 and P40-N50 components that were most affected by alcohol may originate within the primary somatosensory cortex, ${ }^{14-16}$ whereas the amplitude of median N20 and tibial N30 components which may originate from the thalamus, the thalamo-cortical pathways or both, remained unchanged. Our findings are consistent with previous reports that the main effect of alcohol is on the cerebral cortex, although the subcortical structures are also affected. ${ }^{13}{ }^{17-19}$ The suppression of giant
SEPs as well as the improvement of myoclonus, are also found in the therapeutic responses of myoclonus to various drugs. ${ }^{4-7}$

The mechanisms for suppressing both giant SEPs and myoclonus by alcohol remain unclear. It has been postulated that the myoclonic jerks provoked by different external stimuli may be due to an abnormal recruitment and hypersynchronisation of a large population of motor neurons by altered interneuronal inhibitory activity in the sensorimotor cortex. ${ }^{14}$ In addition, the influence by the supplementary motor area and cerebello-thalamic projections to the motor cortex before and during movement may also be exaggerated, leading to complex cortico-subcortical interactions. ${ }^{2021}$ Thus alcohol may exert its effect on myoclonus not only at the sensorimotor cortex but also at other cortical and subcortical areas.

Clonazepam and piracetam may also reduce the giant SEPs and myoclonus. On one occasion, a moderate suppression of amplitude of SEPs were revealed in patients 1 and 2 being treated with clonazepam and piracetam. However, alcohol appears to have a more potent effect in control of action myoclonus than clonazepam or piracetam as suggested by the responses in two of our three patients. Piracetam is particularly effective against cortical reflex myoclonus, perhaps by acting on somatosensory cortex (afferent side). ${ }^{22}$ On the other hand, the alcoholic effect is different from that of primidone, which appears to act on the motor cortex (efferent side) as it considerably improves action myoclonus and reduces reflex myoclonus, but the SEPs usually become large in amplitude. ${ }^{14}$ Although the lesser response to alcohol in the third patient is difficult to explain, the dramatic improvement of myoclonus in two of our three patients encouraged us to give a trial of alcohol in patients with DCM. It also provides us with another pharmacological tool in determining the anatomical-physiological processes which cause myoclonus.

The authors acknowledge the technical assistance of Mrs Fang Fang.

1 Hunt JR. Dyssynergia cerebellaris myoclonica-primary atrophy of the dentate system. Brain 1921;44:490-538. 2 Gilbert GJ, McEntee WJ, Glaser GH. Familial myoclonus and ataxia. Pathophysiologic implications. Neurology

Dawson GD. Investigations on a patient subject to myoclonic seizures after sensory stimulation. J Neurol Neurosurg Psychiatry 1947;10:141-62. 
4 Halliday AM. Cerebral somatosensory and visual evoked potentials in different clinical forms of myoclonus. In: Desmedt JE, ed. Clinical uses of cerebral, brainstem and spinal somatosensory evoked potentials. Basel: Karger 1980:292-310.

5 Chadwick D, Hallett M, Harris R, Jenner P, Reynolds EM, Marsden CD. Manipulation of brain serotonin

6 Fahn S. Postsynaptic action myoclonus: review of the literature and report of two new cases with response to valproate and estrogen. In: Fahn S, Davis JN, Rowland LP, eds. Advances in neurology. New York: Raven Press 1979:49-84.

7 Obeso JA, Artieda J, Rothwell JC, Day B, Thompson P, Marsden $C D$. The treatment of severe action myoclonus. Marsden CD. The treatment of severe action myoclonus.

8 Obeso JA, Rothwell JC, Marsden CD. The spectrum of cortical myoclonus: from focal reflex jerks to spontaneous motor epilepsy. Brain 1985;108:193-22

9 Chu NS. Somatosensory evoked potentials: correlations with height. Electroenceph Clin Neurophysiol 1986;65: 169-76.

10 Marsden CD, Obeso JA. The Ramsay Hunt syndrome is a useful clinical entity. Movement Disorders 1989;4:6-12.

11 Andermann F, Berkovic S, Carpenter S, Andermann E. The Ramsay Hunt syndrome is no longer a useful diagnostic category. Movement Disorders 1989;4:13-7.

12 Harding AE Ramsay Hunt syndrome, Unverricht-Lundborg disease, or what? Movement Disorders 1989;4:18-9.

13 Lewis EG, Dustman RE, Beck ED. The effects of alcohol on visual and somatosensory evoked responses. Electroenceph Clin Neurophysiol 1970;28:202-5.

14 Rothwell JC, Obeso JA, Marsden CD. On the significance of giant somatosensory evoked potentials in cortical myoclonus. J Neurol Neurosurg Psychiatry 1984;47:33-42.

15 Kakigi R, Shibasaki H. Somatosensory evoked potentials following stimulation of the lower limb in cortical reflex myoclonus. J Neurol Neurosurg Psychiatry 1987;50: 1641-6.

16 Kakigi R, Shibasaki H. Generator mechanisms of giant somatosensory evoked potentials in cortical reflex myoclonus. Brain 1987;110:1359-73.

17 Himwich HE, Diperri R, Dravid A, Schweigerdt AK. Comparative susceptibility to alcohol of the cortical area and midbrain reticular formation of the cat. Psychosom Med 1966;28:458-63.

18 Gross MM, Begleiter H, Tobin M, Kissin B. Changes in auditory evoked response induced by alcohol. JNerv Ment Dis 1966:143:152-6.

19 Squires KC, Chu NS, Starr A. Acute effect of alcohol on auditory brainstem potentials in humans. Science 1978; 201:174-6.

20 Halliday AM. The neurophysiology of myoclonic jerking-a reappraisal. In: Charlton $\mathrm{MH}$, ed. Myoclonic Seizures. Amsterdam: Excerpta Medica 1975:1-29.

21 Hallett $M$. The pathophysiology of myoclonus. Trends in Neurosciences 1987;10:69-73.

22 Obeso JA, Artieda J, Quinn NP, et al. Piracetam in the treatment of different type of myoclonus. Clin Neuropharmacol 1988;11:529-36. 